\title{
Ruptured Pseudoaneurysm of the Petrous Internal Carotid Artery Caused by Chronic Otitis Media -Case Report-
}

\author{
Hirofumi OYAma, Kenichi HATTORI, Shigeaki TANAHASHI*, \\ Akira KITO, Hideki MAKI, and Kuniaki TANAHASHI
}

Departments of Neurosurgery and *Otorhinolaryngology, Ogaki Municipal Hospital, Ogaki, Gifu

\begin{abstract}
A 60-year-old man presented with a rare case of pseudoaneurysm in the petrous internal carotid artery (ICA) caused by chronic otitis media manifesting as right otorrhagia after the earpick procedure. Computed tomography showed the destroyed petrous bone; the ICA and the aneurysm, which were exposed to the right middle ear; and a massive hematoma in the right middle ear. Right carotid angiography revealed a pseudoaneurysm arising from the ICA coursing through the middle ear. Two weeks later, the right ICA was trapped after external carotid artery-middle cerebral artery bypass surgery using a vein graft. A biopsy specimen was obtained from the middle ear and the histological diagnosis was inflammatory disease. Pseudoaneurysm often causes sudden massive aural bleeding. The appropriate management strategy may be exclusion of the pseudoaneurysm with revascularization to avoid leaving foreign material in the infected middle ear.
\end{abstract}

Key words: pseudoaneurysm, petrous internal carotid artery, chronic otitis media, otorrhagia, extracranial-intracranial bypass

\section{Introduction}

Pseudoaneurysm of the petrous internal carotid artery (ICA) is rare, and may occur as a result of gunshot penetrating trauma, arterial dissection, invasive tumor, radiation therapy, fibromuscular disease, or as a complication of surgery. ${ }^{1-16)}$ Such pseudoaneurysms may grow and become a potential source of thromboembolic complications or rupture. ${ }^{1-4)}$ Pseudoaneurysm caused by infectious disease such as chronic otitis media is extremely rare, with only six reported cases. ${ }^{2,4,7,8,10,15)}$ We treated a rare case of ruptured pseudoaneurysm in the petrous ICA caused by chronic otitis media.

\section{Case Report}

A 60-year-old man developed repeated hemorrhages after undergoing the earpick procedure. He presented with acute massive hemorrhage from the right external auditory meatus and was admitted to the Department of Otorhinolaryngology. Fresh blood (50 ml) flowed out during a short period and hemostasis was accomplished with difficulty using compression with oxycellulose and cotton balls. Release of the compress resulted in relapse of the bleeding. He had no history of trauma, surgery of the head and neck, or irradiation.

Computed tomography with contrast medium showed

Received September 8, 2009;

Accepted December 24, 2009 the destroyed petrous bone; the ICA and the aneurysm, which were exposed to the right middle ear; and a massive hematoma in the right middle ear (Fig. 1). Right carotid angiography revealed a pseudoaneurysm arising from the right ICA coursing through the middle ear (Fig. 2). The bony structure around the ICA was destroyed around the middle ear, although no enhanced tumor was identified. Therefore, we believe that the pseudoaneurysm was caused by chronic otitis media, rather than by simple dissection and a tumor.

Two weeks after the onset of symptoms, the right ICA was trapped. Coil embolization of the distal ICA was performed under guidance from intraoperative angiography. The proximal ICA was then ligated. External carotid ar-
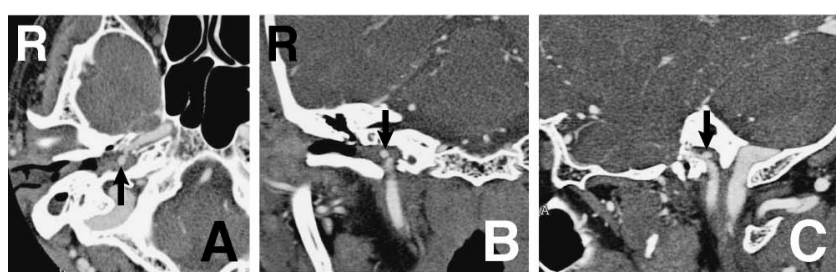

Fig. 1 Axial (A), coronal (B), and sagittal (C) computed tomography scans with contrast medium showing the destroyed petrous bone, the internal carotid artery and the aneurysm (arrow), which are exposed to the right middle ear, and a massive hematoma in the right middle ear. 

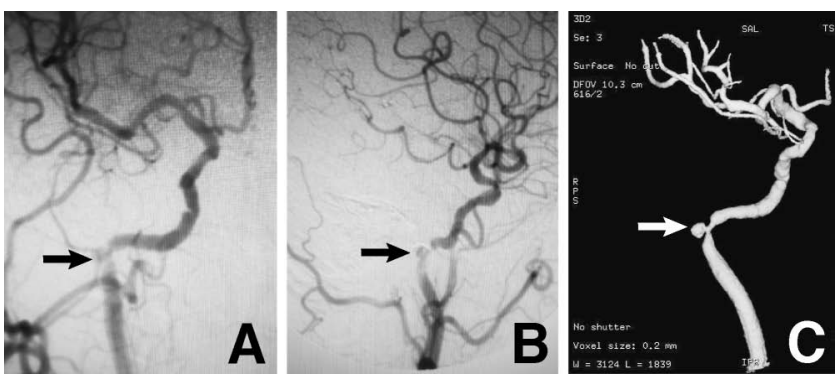

Fig. 2 Right carotid angiograms, anteroposterior (A), lateral (B), and three-dimensional oblique views (C), showing a pseudoaneurysm (arrow) arising from the right internal carotid artery coursing through the middle ear.
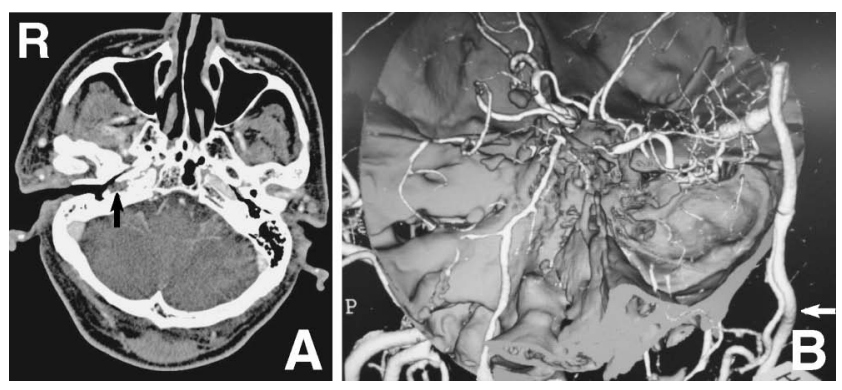

Fig. 3 (A) Postoperative computed tomography scan with contrast medium showing complete obliteration of the right internal carotid artery and aneurysm (arrow), and disappearance of the hematoma from the right middle ear. (B) Postoperative three-dimensional computed tomography angiogram demonstrating the patent vein graft (arrow) between the right external carotid and middle cerebral arteries.

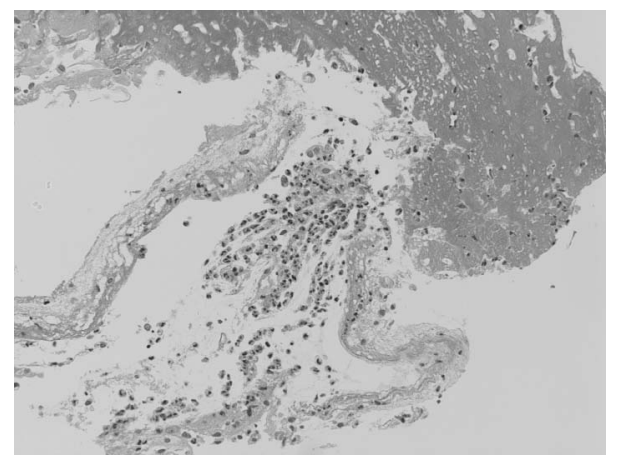

Fig. 4 Photomicrograph showing neutrophils and lymphocytes among the stratified squamous epithelium and the hematoma. The histological diagnosis is inflammatory disease. Hematoxylin-eosin stain, original magnification $\times 200$.

tery-middle cerebral artery bypass surgery was also performed using a vein graft in parallel. Postoperative computed tomography with contrast medium showed complete obliteration of the right ICA and aneurysm (Fig. 3A). The hematoma also disappeared from the right middle ear.
Three-dimensional computed tomography angiography confirmed a patent vein graft (Fig. 3B).

Eleven days later, biopsy of the middle ear lesion was performed through the eardrum, which had already been perforated. The middle ear cavity had become clean by this time. Neutrophils and lymphocytes were found among the stratified squamous epithelium and the hematoma. The histological diagnosis was inflammatory disease (Fig. 4). Audiometry before discharge showed deafness in the right ear.

\section{Discussion}

The previous cases of pseudoaneurysm in the petrous ICA caused by chronic otitis media manifested as facial numbness, diplopia, seventh cranial nerve dysfunction, and auditory dysfunction, ${ }^{5,7,10,11)}$ whereas dysfunction of the ninth, tenth, and twelfth cranial nerves was less common. Direct pressure of the pseudoaneurysm on these nerves caused disturbance in nerve function. Pain related to eustachian tube blockage by the aneurysm may occur. ${ }^{16)}$ Pseudoaneurysms can also cause pulsatile tinnitus or bruit, and are sometimes discovered as a retrotympanic vascular mass during otological examination. ${ }^{5,7,10,11)}$ Pseudoaneurysms can also manifest as massive otorrhagia or epistaxis following acute rupture, so these rare aneurysms require care. ${ }^{1,2,4,10,16)}$

Our patient did not identify hearing disturbance before admission. However, we suspected that chronic otitis media had already caused perforation of the eardrum. The stick for the earpick procedure is intended to enter into the middle ear through a perforation in the eardrum. Based on the amount of petrous bone destruction around the ICA, we believe that the inflammation from chronic otitis media had induced the pseudoaneurysm. The stick for the earpick procedure probably scratched the pseudoaneurysm and caused massive otorrhagia. Therefore, the earpick procedure, which requires blind manipulation, may be very risky in a patient with chronic otitis media.

Our patient presented with only otorrhagia. However, rupture of the pseudoaneurysm could have caused epistaxis through the eustachian tube. Direct compression may not be able to stop epistaxis, and aspiration of the blood may cause apnea. In that situation, a patient may enter a critical condition, following hemorrhagic shock or respiratory failure.

The appropriate management strategy for these rare lesions is unclear. ${ }^{6,14)}$ Revascularization of the carotid artery, using a cervical to middle cerebral artery vein bypass graft, may be necessary to provide immediate restoration of high blood flow, and to reduce the risk of morbidity and mortality caused by acute ischemic complications.

There are five reported cases of a ruptured pseudoaneurysm caused by chronic otitis media. Three cases were treated without revascularization and one case was treated with revascularization as in our patient. ${ }^{2,4,8,10)}$ Coil embolization of the aneurysm dome was reported in one patient. However, we did not select this method to avoid leaving foreign material in the infected middle ear. ${ }^{7)}$ Treatment with a self-expandable covered stent passing through 
the pseudoaneurysm of the petrous carotid segment has only been used in one case of unruptured pseudoaneurysm resulting from carotid fibromuscular dysplasia. $^{3)}$

Pseudoaneurysm often causes sudden massive aural bleeding. The appropriate management strategy may be exclusion of the pseudoaneurysm with revascularization to avoid leaving foreign material in the infected middle ear.

\section{References}

1) Auyeung KM, Lui WM, Chow LC, Chan FL: Massive epistaxis related to petrous carotid artery pseudoaneurysm after radiation therapy: emergency treatment with covered stent in two cases. AJNR Am J Neuroradiol 24: 1449-1452, 2003

2) Chiappetta F, Vangelista S, Pirrone R: Recurrent massive otorrhagia caused by a petrous carotid aneurysm. J Neurosurg Sci 26: 205-207, 1982

3) Cohen JE, Grigoriadis S, Gomori JM: Petrous carotid artery pseudoaneurysm in bilateral carotid fibromuscular dysplasia: treatment by means of self-expanding covered stent. Surg Neurol 68: 216-220, 2007

4) Costantino PD, Russell E, Reisch D, Breit RA, Hart C: Ruptured petrous carotid aneurysm presenting with otorrhagia and epistaxis. Am J Otol 12: 378-383, 1991

5) Halbach VV, Higashida RT, Hieshima GB, Dowd CF, Barnwell SL, Edwards MS, Melicharek M: Aneurysms of the petrous portion of the internal carotid artery: results of treatment with endovascular or surgical occlusion. AJNR Am J Neuroradiol 11: 253-257, 1990

6) Henriksen SD, Kindt MW, Pedersen CB, Nepper-Rasmussen HJ: Pseudoaneurysm of a lateral internal carotid artery in the middle ear. Int J Pediatr Otorhinolaryngol 52: 163-167, 2000

7) Kawakami K, Kayama T, Kondo R, Kureyama H, Maruya J, Nakai O, Hosoya T, Yamaguchi K: [A case of mycotic ICA petrous portion aneurysm treated with endovascular surgery]. No Shinkei Geka 24: 253-257, 1996 (Japanese)

8) Kimmelman CP, Grossman R: Intratemporal carotid aneurysm as a complication of chronic otitis media: treatment with balloon catheter obliteration. Otolaryngol Head Neck Surg 91: 306-308, 1983

9) Kudo S, Colley DP: Multiple intrapetrous aneurysms of the internal carotid artery. AJNR Am J Neuroradiol 4: 11191121,1983

10) McGrail KM, Heros RC, Debrun G, Beyerl BD: Aneurysm of the ICA petrous segment treated by balloon entrapment after EC-IC bypass. Case report. J Neurosurg 65: 249-252, 1986

11) Morantz RA, Kirchner FR, Kishore P: Aneurysms of the petrous portion of the internal carotid artery. Surg Neurol 6: 313-318, 1976

12) Oates JW, McAuliffe W, Coates HL: Management of pseudoaneurysm of a lateral aberrant internal carotid artery. Int J Pediatr Otorhinolaryngol 42: 73-79, 1997

13) Rawlinson J, Colquhoun IR: Aneurysms involving the intrapetrous internal carotid artery: a rare cause of Horner's syndrome. Br J Radiol 63: 69-72, 1990

14) Rostomily RC, Newell DW, Grady MS, Wallace S, Nicholls S, Winn HR: Gunshot wounds of the internal carotid artery at the skull base: management with vein bypass grafts and a review of the literature. J Trauma 42: 123-132, 1997

15) Samuel J, Fernandes CM: Mycotic aneurysm of the petrous portion of the internal carotid artery. J Laryngol Otol 103: 111-114, 1989

16) Willinsky R, Lasjaunias $P$, Pruvost $P$, Boucherat M: Petrous internal carotid aneurysm causing epistaxis: balloon embolization with preservation of the parent vessel. Neuroradiology 29: 570-572, 1987

Address reprint requests to: Hirofumi Oyama, M.D., Department of Neurosurgery, Ogaki Municipal Hospital, 4-86 Minamikawa-machi, Ogaki, Gifu 503-8502, Japan.

e-mail: oya3776@arrow.ocn.ne.jp 